\title{
Search for Dark Matter with Heavy Quarks and Jets Using the CMS Detector
}

\section{Tommaso Dorigo*}

INFN, Sezione di Padova

E-mail: dorigo@pd.infn.it

This document summarizes results of searches for dark matter produced in association with topquark pairs, and for vector and axial-vector dark matter mediators in final states with pairs of high-mass hadronic jets. The searches have been performed by the CMS collaboration in 13-TeV proton-proton collisions data delivered to the detector in 2016 by the Large Hadron Collider at CERN.

2nd World Summit: Exploring the Dark Side of the Universe 25-29 June, 2018

University of Antilles, Pointe-à-Pitre, Guadeloupe, France

${ }^{*}$ Speaker. 


\section{Introduction}

The CMS collaboration performed a number of searches for dark matter(DM) in the large dataset of $\sqrt{s}=13 \mathrm{TeV}$ proton-proton collisions delivered by the Large Hadron Collider (LHC) since 2015, in its Run 2 data-taking period. In this document we summarize the results of some of them, carried out in data collected during 2016 and corresponding to a total integrated luminosity of up to $35.9 \mathrm{fb}^{-1}$.

Dark matter particles produced in hadronic collisions, while not interacting with the detector in a directly measurable way, may still leave a trace of their production in the form of a significant overall imbalance of the transverse momentum of visible particles. This well-known and routinely exploited signature can be combined with the one yielded by the decay of other particles that may be produced in association with the dark matter, enhancing the experimental sensitivity. Such a strategy is the one described in Sec.2, where results of a search for DM in association with topquark pairs in data collected by CMS in 2016[1] are presented.

An indirect way to cast light on the possible particle explanation of DM in the universe is to test new physics models that, besides the DM particle, predict the existence of a heavy vector or axial-vector mediator, a spin-1 particle that mediates interactions between quarks and DM, and which may produce both DM or hadronic jets in its decays. Searches for a generic dijet-decaying resonances of high invariant mass are sensitive to this scenario. The corresponding results produced by CMS in the analysis of 2016 data[2] are discussed in Sec.3.

Two additional methods to search for dark matter mediators have been exploited by CMS. The first[3] is to search for deviations of the angular distribution of high-mass jet pairs from QCD predictions, as the inclusion of the DM mediator would affect it in a measurable way. Results of this approach are detailed in Sec.4. In the second method[4] the search for a heavy mediator can be performed in events where a hadronic jet produced by initial state radiation, and emitted at large transverse momentum, recoils against a "fat jet" resulting from the decay of a strongly boosted DM mediator. The recent improvements in the technique of jet substructure allow this topology to be a sensitive probe of the high- $p_{T}$ production of dijet-decaying new particles. The results of such a technique in the context of a DM mediator search are described in Sec.5.

\section{Search for Dark Matter with Top Quark Pairs}

The absence of new physics at the energy scale currently probed by the LHC experiments may indicate the correctness of the hypothesis called "Minimal Flavor Violation" [5, 6]. In this scenario, a new interaction which mediates the interaction of dark matter with ordinary standard model (SM) particles is carried by a mediator which inherits the Yukawa structure of the SM. This may enhance the coupling to DM of top quarks. The associated production of top quark pairs and pairs of DM particles thus offers itself as a proficuous means of testing this hypothesis. CMS has sought for the signature of a top quark pair associated with a large missing transverse energy produced by the escaping DM particle pair, in events where the top quarks both decay semileptonically[1]. 


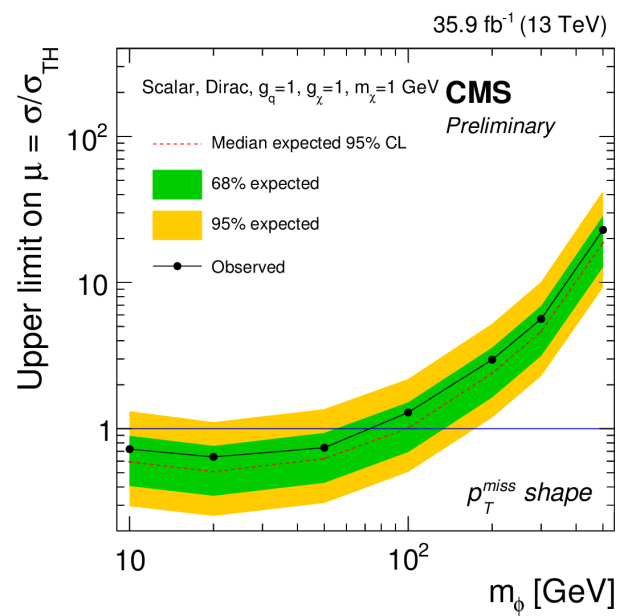

(a) $p_{\mathrm{T}}^{\text {miss }}$ shape: scalar mediator

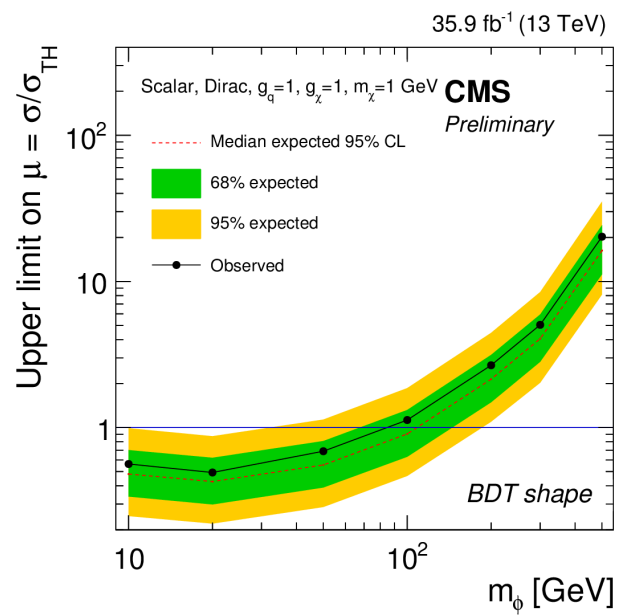

(c) BDT discriminant: scalar mediator

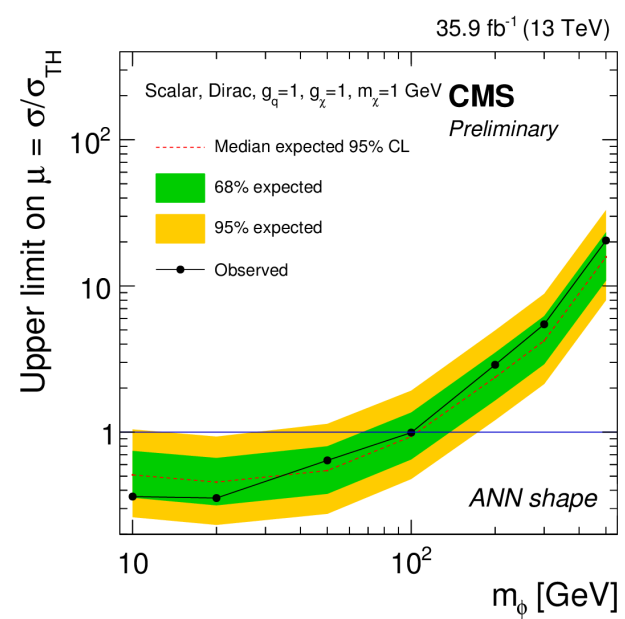

(e) ANN discriminant: scalar mediator

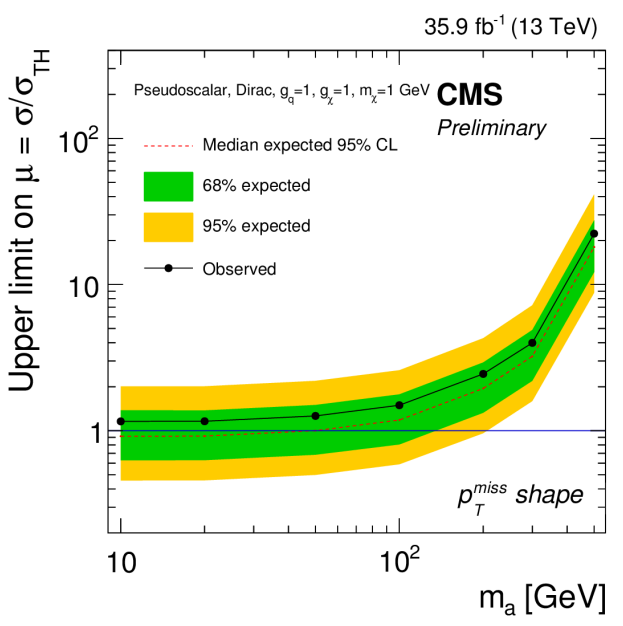

(b) $p_{\mathrm{T}}^{\text {miss }}$ shape: pseudoscalar mediator

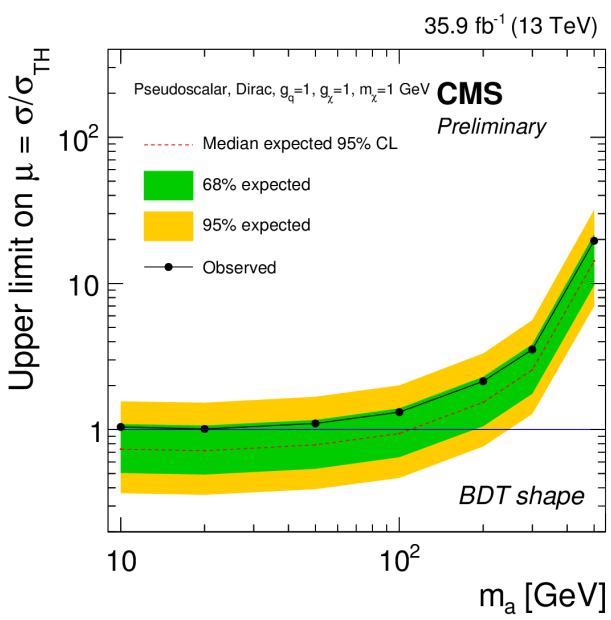

(d) BDT discriminant: pseudoscalar mediator

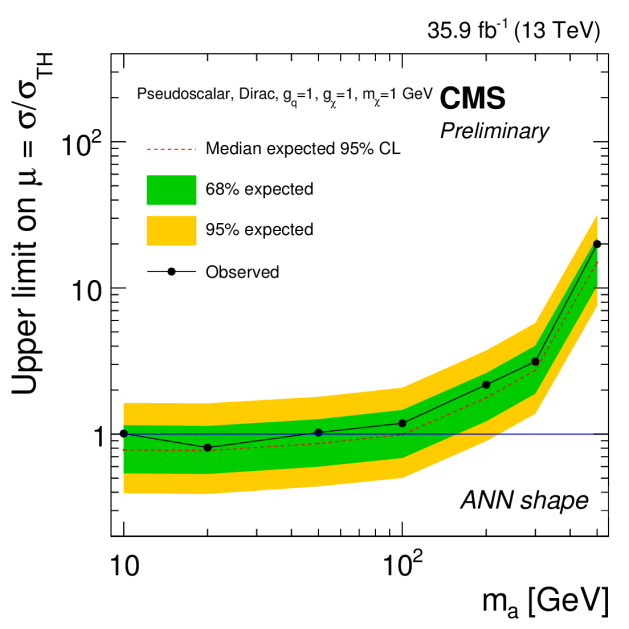

(f) ANN discriminant: pseudoscalar mediator

Figure 1: Upper limits on the signal strength, in units of the theoretically predicted cross section, for scalar (left) and pseudo-scalar mediators (right) obtained by the three separate search techniques described in the text. The green (yellow) bands bracket $15.9 \%$ and $84.1 \%$ (2.2\% and 97.8\%) quantiles of the expected upper limit, and the black curves show the observed $95 \%$ C.L. upper limit as a function of the mediator mass. 
In the simplified scenario considered by the CMS search, the interaction is flavor-universal $\left(g_{q}=1\right)$, and the DM particle mass is set at $1 \mathrm{GeV}$. The mediator-new fermion interaction is also governed by a coupling set to $g_{\chi}=1$. Three separate strategies are employed to put in evidence the signal in the data, where backgrounds are dominated by SM top-quark pair production. The first strategy extracts information directly from the shape of the missing transverse momentum distribution; the second employs a boosted decision tree discriminant; and the third uses the output of an artificial neural network.

The three techniques extract 95\% C.L. upper limits on the signal strength modifier $\mu$, defined as the signal cross section calculated in units of the theoretical prediction, $\mu=\sigma / \sigma_{T H}$. Results are extracted separately for the case of a scalar and a pseudoscalar mediator, as a function of the mediator mass (see Fig.1). The observed (expected) 95\% CL exclusions for a scalar mediator are $m_{\phi}<74(99) \mathrm{GeV}$. The expected exclusion for a pseudoscalar mediator is $m_{a}<50 \mathrm{GeV}$, while no exclusion is set by the observed data.

\section{Search for DM Mediators in Jet Pairs}

A number of new physics models can be tested at the LHC by searching for narrow resonances that decay into jet pairs. The signal may appear as a resolution-shaped Gaussian-like bump, deformed by final state radiation effects, in the otherwise smooth distribution of the large background from QCD $2 \rightarrow 2$ processes. CMS searched for such a signal in 2016 data collected by two different strategies: a normal trigger selecting high-mass jet pairs, and a "data scouting" trigger path that allows the collection of lower-mass, higher-rate processes by restricting the information saved per each event[2]. In the first case the signal is sought for invariant masses above $1.6 \mathrm{TeV}$, while in
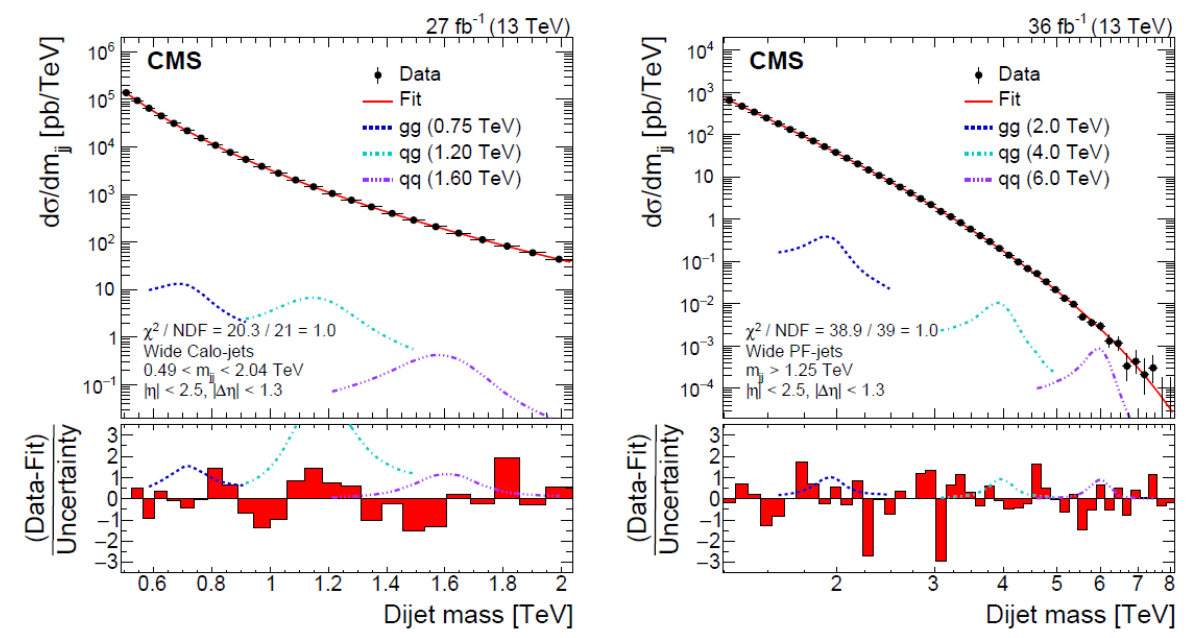

Figure 2: Background-only fits to data in the low-mass (left) and high-mass search (right). Some representative signal shapes from resonances decaying to quark-antiquark, quark-gluon, and gluon pairs are overlaid for comparison. The lower panels show the deviations of data from the fit in units of the statistical uncertainty of the data.

the second case the lower-mass range $0.6-1.6 \mathrm{TeV}$ can be accessed. The data correspond to an integrated luminosity of $35.9 \mathrm{fb}^{-1}$ and $27 \mathrm{fb}^{-1}$ of $13-\mathrm{TeV}$ collisions acquired in 2016 , respectively. 
In the context of DM searches, the scenario considered for the search of a spin-1 vector or axialvector mediator in the dijet mass spectrum is a simplified one [7, 8] where the mediator is leptophobic, and can thus decay only to quark pairs or to DM pairs. The DM particles are assumed to have a mass of $1 \mathrm{GeV}$, and have a universal quark coupling $g_{q}=0.25$ and a DM coupling set to $g_{D M}=1.0$.

Data histograms of the smooth reconstructed dijet mass spectrum are fit in both the low-mass and the high-mass search to QCD-inspired functional forms; for the low-mass search five parameters are necessary to interpret the shape of the data, while for the high-mass search a four-parameter function already used in previous searches is found sufficient. The data and the background-only fits are shown in Fig.2.
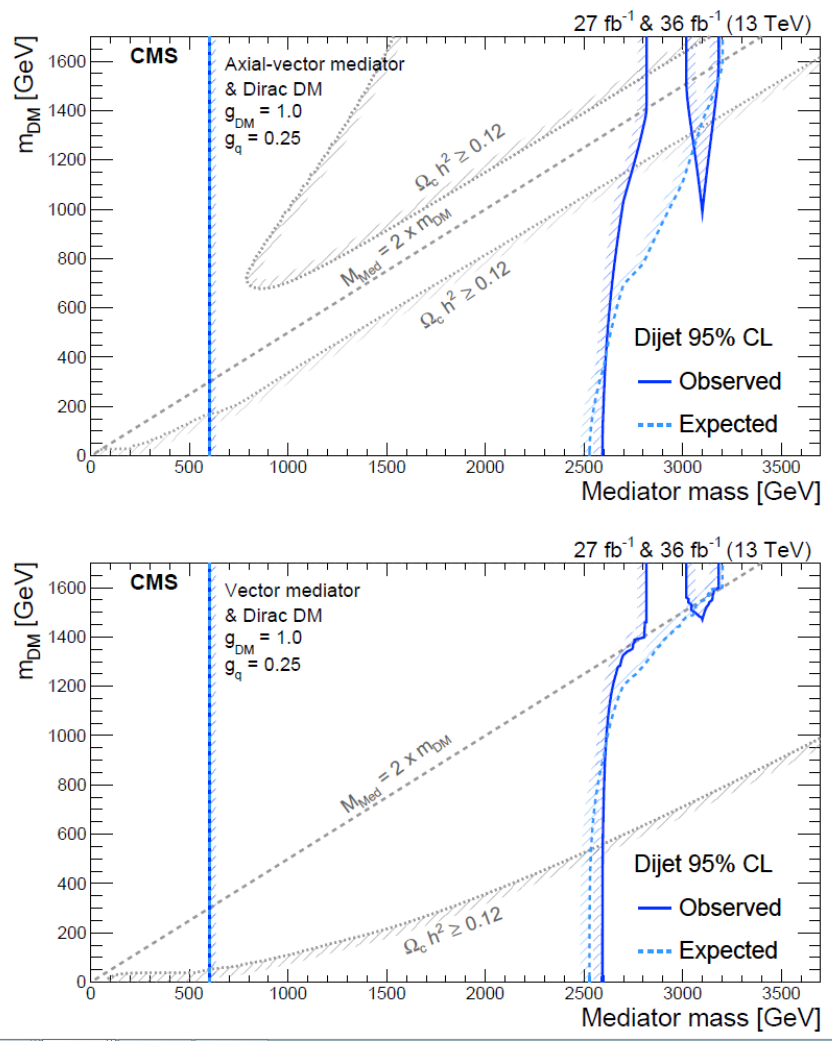

Figure 3: Expected (dashed) and observed (solid) exclusion regions, at 95\% C.L., in the plane of DM mass as a function of mediator mass for axial-vector (upper panel) and vector mediator (lower panel). Also shown are regions of parameter space where the abundance of DM exceeds the cosmological relic density[9] (light gray). The excluded regions are valid for Dirac DM and for a universal quark coupling $g_{q}=0.25$ and a DM coupling $g_{D M}=1.0$.

The absence of a significant signal in the data can be converted in a 95\% C.L. upper limit on the allowed regions of the model parameters, namely the mediator mass versus the mass of the DM particle. The excluded regions are shown in Fig.3.

\section{Search for DM Mediators in Jet Angular Distributions}

While QCD processes produce jet pairs with a flat distribution of the kinematical variable $\chi=$ 
$\exp \left(\left|y_{1}-y_{2}\right|\right)$, where $y_{i}$ are the rapidities of the final state jets, new massive particles such as a DM mediator will preferentially yield in their decay pairs of jets with low values of $\chi$. This difference has been exploited by a new CMS analysis[3] that directly studied the distribution of $\chi$ in seven different ranges of the dijet invariant mass. The study of the dijet angular distribution is more sensitive than a direct resonance search such as the one reported in Sec.3 for large values (above 0.4 ) of the universal quark coupling parameter $g_{q}$, when the narrow-width approximation fails and the resonance shape becomes less easy to distinguish from backgrounds. The study reported here used the full statistics of 2016 proton-proton collisions, corresponding to an integrated luminosity of $35.9 \mathrm{fb}^{-1}$.

Fits to the $\chi$ distributions are used to extract 95\% C.L. limits on the value of $g_{q}$ as a function of DM mediator mass. An excess corresponding to 2.8 standard deviations is present for mediator masses between 4.5 and $6 \mathrm{TeV}$, corresponding to the black line showing the observed limit ranging higher than the yellow band in Fig.4.

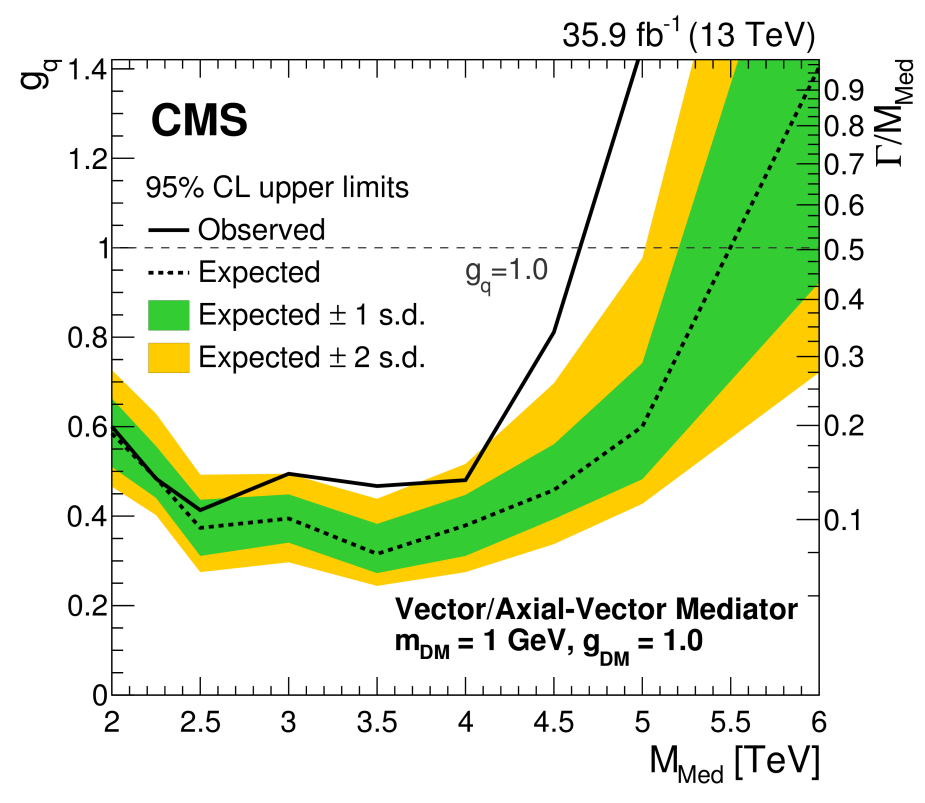

Figure 4: The black line shows observed 95\% CL upper limits on the quark coupling $g_{q}$ as a function of the DM mediator mass, for the case of an axial-vector or vector DM mediator with $g_{D M}=1.0$ and a dark matter particle mass $m_{D M}=1 \mathrm{GeV}$. Green and yellow bands indicate 1- and 2- $\sigma$ variations in the expected limit. The dotted horizontal line shows the coupling strength of a benchmark DM mediator with $g_{q}=1.0$. The corresponding limits on the natural width of the DM mediators are shown on the vertical axis on the right-hand side of the figure.

\section{Search for DM Mediators in Boosted Jets}

The signal of a low-mass $Z^{\prime}$ boson decaying to jet pairs has been sought in data collected in 2016 by the CMS experiment, and corresponding to an integrated luminosity of $35.9 \mathrm{fb}^{-1}$, using a novel technique that exploits the recoil of the putative new boson off a high-transverse-momentum jet produced by initial-state radiation. In that case, the decay products of the $Z^{\prime}$ are collected within a wide 
hadronic jet, and the soft-drop jet substructure technique $[10,11]$ can be employed to disentangle the hard final state partons from soft radiation and reconstruct the resonance mass. This allows to strongly reduce the QCD background and to evidence the reference signal of hadronically-decaying $W$ and $Z$ bosons, as shown in Fig.5.

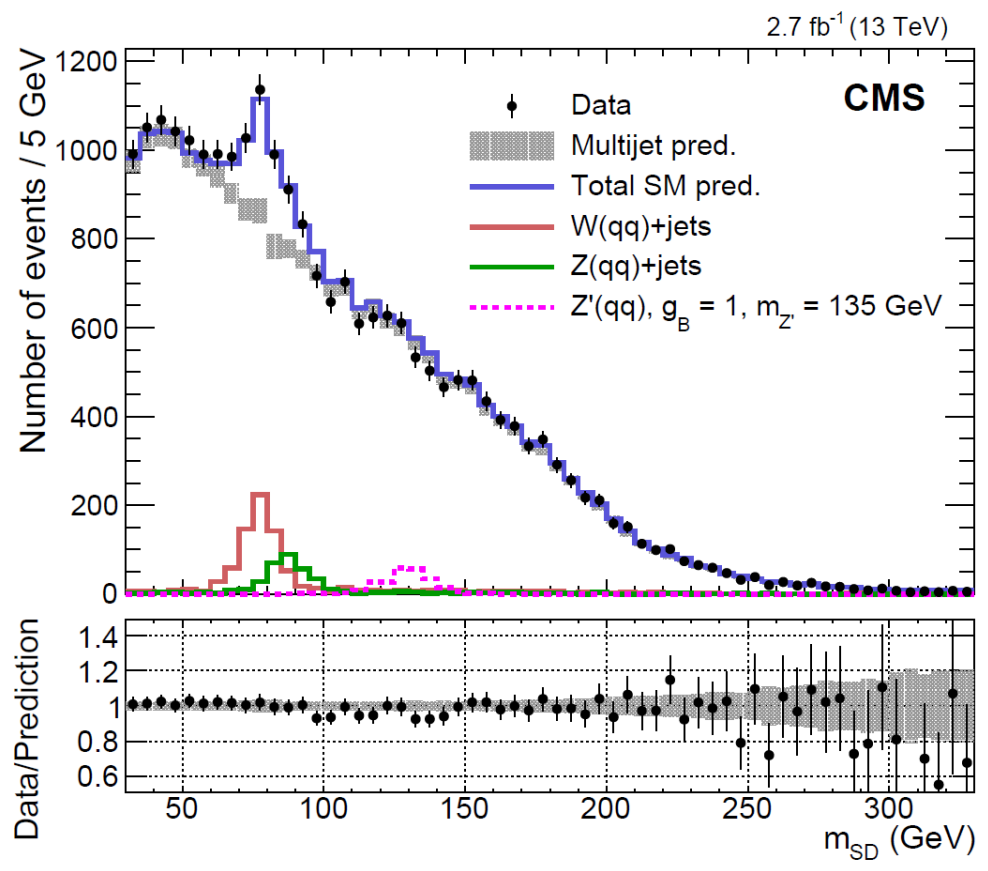

Figure 5: Distribution of the jet mass computed with the soft drop algorithm in CMS data after the final selection. The contribution of $W$ and $Z$ boson decays are shown, as well as the one from a hypothetical $Z^{\prime}$ boson with mass of $135 \mathrm{GeV}$. The ratio between observed data and background prediction is shown in the bottom panel.

The absence of a signal in the reconstructed mass distribution has been interpreted in the context of a simplified model with a leptophobic vector DM mediator and assuming $g_{q}=0.25$ and $g_{D M}=1$. Figure 6 shows the excluded regions in the plane of DM mass versus mediator mass.

\section{Conclusions}

This report summarizes searches for new physics performed by the CMS experiment using 2016 proton-proton collisions data at $\sqrt{s}=13 \mathrm{TeV}$ from Run 2 of the LHC. The simplified theoretical models considered in the searches include scalar or pseudo-scalar dark matter mediators produced in association with top quark pairs, as well as vector or axial-vector mediators produced directly and decaying to high-mass jet pairs or low-mass jet pairs with high transverse boost. None of the above searches evidence any signal, and their results are used to set limits on the model parameters. A study of angular distributions of jet pairs finds a mild excess of data at low values of the angular variable $\chi$ for DM mediator masses above $4.5 \mathrm{TeV}$. 


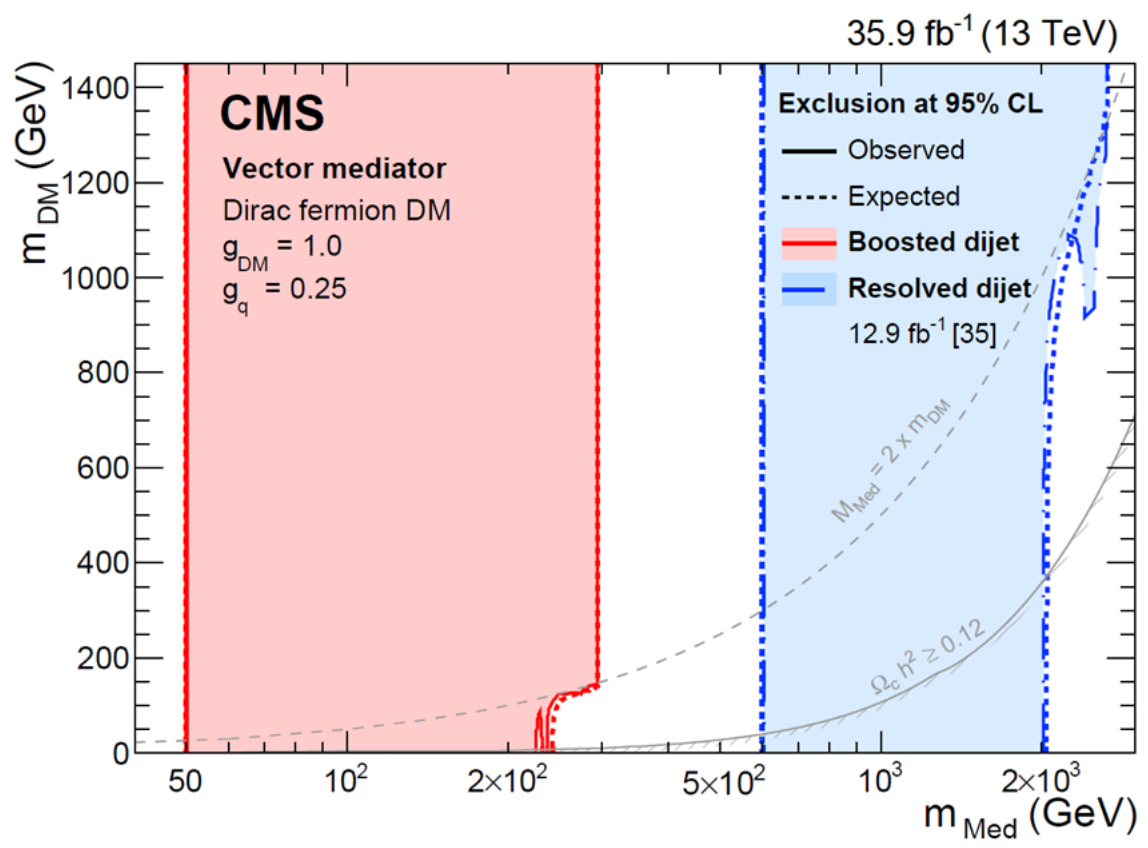

Figure 6: Exclusion region in the plane of DM particle mass versus vector mediator mass obtained by the analysis of boosted jet pairs described in the text (area in light red). For comparison are shown the results of a resolved jet pair search [12] (in light blue) and the constraints from cosmological relic density of DM (light gray) determined as described in [13] from astrophysical measurements [14, 15].

\section{References}

[1] CMS Collaboration, CMS PAS EXO-17-014 (2018).

[2] CMS Collaboration, Jour. High En. Ph. 08 (2018) 130.

[3] CMS Collaboration, Eur. Phys. Journ. C 78 (2018) 789.

[4] CMS Collaboration, J. High En. Ph. 01 (2018) 097.

[5] G. D’Ambrosio, G. Giudice, G. Isidori, and A. Strumia, Nucl. Phys. B 645 (2002) 155.

[6] G. Isidori and D. M. Straub, Eur. Phys. J. C 72 (2012) 2103.

[7] A. Boveia et al., arxiv:1603.04156 (2016).

[8] J. Abdallah et al., Phys. Dark Univ. 9-10 (2015) 8.

[9] Planck Collaboration, Astron. Astrophys. 594 (2016) A13.

[10] M. Dasgupta et al., J. High En. Ph. 09 (2013) 029.

[11] A. J. Larkoski et al., J. High En. Ph, 05 (2014) 146.

[12] CMS Collaboration, Phys. Lett. B 769 (2017) 520.

[13] T. du Pree, K. Hahn, P. Harris, and C. Roskas, arXiv:1603.08525 (2016).

[14] WMAP Collaboration, Astrophys. J. Suppl. 170 (2007) 377.

[15] Planck Collaboration, Astron. Astrophys. 571 (2014) A16. 